\title{
DETECTION AND SEGMENTATION OF FMCW RADAR SIGNALS BASED ON THE CHIRPLET TRANSFORM
}

\author{
Fabien Millioz and Michael E. Davies \\ School of Engineering and Electronics \\ The University of Edinburgh, King's Buildings, Mayfield Rd \\ Edinburgh EH9 3JL - United Kingdom \\ email: $\{$ fmillioz,mike.davies $\} @$ ed.ac.uk
}

\begin{abstract}
In this paper we present a algorithm designed to detect and characterise the signal coming from Frequency Modulation Continuous Wave radars. The signals are made of linear frequency modulations. A few relevant coefficients of the chirplet transform are selected, and then gathered into chirps whose starting time, length, and chirprate are estimated. An example is provided on a synthetic signal.
\end{abstract}

Index Terms - LPI radar, FMCW radar, chirplet transform, parameter estimation

\section{INTRODUCTION}

Low Probability of Intercept (LPI) Radars are a type of radar designed to hide their emissions from hostile receivers. It aims to see without being seen, which is critical on battlefields. This goal may be achieved by several techniques, such as power management, antenna side lobe reduction or frequency agility [1].

This paper aims to detect and identify Frequency Modulation Continuous Wave (FMCW) radars. This class of LPI radar signals may be modelled by

$$
r(t)=\sum_{n=1}^{N} A_{n} \cos \left(2 \pi f_{n}(t) t+\phi_{n}\right),
$$

with $f_{n}(t)$ a piecewise linear function and $n(t)$ a white Gaussian noise of variance $\sigma^{2}$. To extend the possibility of the model, we consider both continuous and non-continuous functions $f_{n}(t)$. In the context of a radar interceptor, a sensor should detect a signal $s(t)$ coming from several LPI radars, embedded in a white Gaussian noise $n(t)$ of variance $\sigma^{2}$

$$
s(t)=n(t)+\sum_{i=1}^{I} r_{i}(t)
$$

This work was supported by the EPSRC [Grant Number EP/H012897/1], the MOD University Defence Research Centre on Signal Processin and the European Commission through the SMALL project under FET-Open, grant number 225913. where $I$ radars emits the signals $r_{i}(t)$.

Given the model, the chirplet transform [2] seems to be an natural tool to analyse the signal. Several methods exist based on the chirplet transform.

Matching-pursuit based method [3] by Leveau et al., which searches for chirps in the signal and iteratively subtract them from the signal. The main drawback is the computations required by the matching-pursuit framework. Chirplet chains $[4,5]$ are based on the search of a single best path in the time-frequency domain, and are applied in the low Signalto-Noise Ration (SNR) context of gravitational waves. Only a non-linear chirp is detected in the signal. A more general chirplet chain based on a parametric model is proposed by Dugnal et al. [6], which uses local maxima to start chirplet chains, and a single criterion based on the smoothness of the frequency modulation. In our case, we have a strong a priori information used in these works, the chirps are piece-wise linear, and in a multi-signal case, chirps may be crossing.

This paper proposes a new algorithm for detecting and estimating the parameters of the chirps constituting FMCW radars. This method is based on the chirplet transform, presented in the section 2. The main goal is to amalgamate chirplet coming from a crude chirplet transform into different chirps, corresponding to the instantaneous frequency lines $f_{n}(t)$ from the model 1 . To do this, section 3 presents a selection of relevant chirplet coefficients. This selection is comes first from a signal detection signal with an estimated noise level. To have as few relevant coefficients as possible, a refining of this detection is made with a search for local maxima. These coefficients are gathered in an algorithm presented in section 4, and used to estimate the chirps parameters. A result of this algorithm is given on a synthetic signal imitating an antenna receiving four FMCW radar signals.

\section{DISCRETE CHIRPLET TRANSFORM}

The chirplet transform [2] $C(n, k, c)$ of a discrete signal $x[m]$ is determined by 


$$
C(n, k, c)=\sum_{m=-\frac{M_{\phi}-1}{2}}^{+\frac{M_{\phi}-1}{2}} x[n+m] \phi[m] e^{j 2 \pi \frac{c}{2} m^{2}} e^{-j 2 \pi m \frac{k}{K}}
$$

with $n, k$ are the time and frequency indices respectively, $\phi[m]$ an energy-normalised smoothing window of $M_{\phi}$ points, $K$ the number of frequencies and $l$ the chirprate. Let $N$ be the number of time indices.

The chirprate is discretized such that

$$
c=\frac{l}{L} c_{\max }
$$

with $l$ an integer varying from $-L$ to $L$. Consequently, there are $2 L+1$ different chirprates.

The chirplet transform may be interpreted twofold. By considering a chirped window $\phi_{c}[m]=\phi[m] e^{j 2 \pi \frac{c}{2} m^{2}}$, the chirplet transform has the same definition as the usual Short Time Fourier Transform, using a Fast Fourier Transform algorithm. On the other hand, it may be interpreted as the projection of the signal $x[m]$ on a set of atoms $e^{-j 2 \pi\left(\frac{k}{K} m+\frac{c}{2} m^{2}\right)}$, corresponding to chirplets of different chirprates $c$, centred on time $n$ and normalised frequency $\frac{k}{K}$.

The chirplet is redundant: the redundancy coming from the usual STFT is multiplied by the number of chirprates. Next section aims to select a set of relevant chirplet coefficients to use for the estimation of the chirps' parameters.

\section{SIGNAL DETECTION}

The goal of this section is to select a set of chirplet coefficients relevant to the chirps to estimate. Additionally, we try to select as few coefficients as possible, to limit the computation needed for the estimation.

A first step in the selection of chirplet coefficients is to determine whether a time-frequency coefficient contains signal, called hypothesis $\mathcal{H}_{1}$, or not, the hypothesis $\mathcal{H}_{0}$. We consider the square modulus of the chirplet transform, the energy of the chirplet coefficients. A chirplet coefficient containing signal should have a higher energy than coefficients containing noise only. In other words, the hypothesis test is solved by selecting a detection threshold $t$.

In the hypothesis $\mathcal{H}_{0}$, the chirplet coefficient contains Gaussian noise only, and consequently has a circular complex Gaussian distribution, and its square modulus has a $\chi^{2}$ distribution with two degrees of freedom.

To simplify the detection problem, we define the maximum chirplet $D[n, k]$ at a given time-frequency point $[n, k]$, containing all maxima of the square modulus of the chirplet coefficients along the chirprates

$$
D[n, k]=\max _{c}|C[n, k, c]|^{2} .
$$

The chirprates $C R[n, k]$ associated to the maxima are

$$
C R[n, k]=c /|C[n, k, c]|^{2}=D[n, k] .
$$

In the hypothesis $\mathcal{H}_{0}, D[n, k]$ is the maximum of $2 L+1$ random variables having a $\chi^{2}$ distribution with two degrees of freedom. Assuming that $\left|C\left[n, k, c_{i}\right]\right|^{2}$ and $\left|C\left[n, k, c_{j}\right]\right|^{2}$ for $i \neq j$, the order statistics [7] leads to the distribution of $D[n, k]$ of noise only $f_{D[n, k], \mathcal{H}_{0}}$

$$
f_{D[n, k], \mathcal{H}_{0}}=(2 L+1)\left(1-e^{-\frac{-x}{2 \sigma^{2}}}\right)^{2 L} \frac{1}{2 \sigma^{2}} e^{-\frac{-x}{2 \sigma^{2}}}
$$

In this paper, the detection strategy chosen is a NeymanPearson approach. With a chosen probability of false alarm $p_{f a}$, the threshold $t$ is such that

$$
\begin{gathered}
t / \operatorname{Prob}\left(D[n, k]_{\mathcal{H}_{0}}>t\right)=p_{f a} \\
t=-\sigma^{2} \ln \left(1-\left(1-p_{f a}\right)^{\frac{1}{2 L+1}}\right)
\end{gathered}
$$

This threshold depends on the noise level $\sigma^{2}$, which must be estimated. To do this, we use a noise level estimation based on the spectral kurtosis of the minimal statistics [8]. This estimation is not discussed in this paper, and we consider in the following that the noise level is known.

We aim to detect only a few signal points, consequently we choose a low probability of false alarm, that is $p_{f a}=$ $10^{-5}$. The set of detected points $\mathcal{S}$ is

$$
\mathcal{S}=\{[n, k] / D[n, k]>t\}
$$

This set contains the maxima of the energy of the signal in the time-frequency plane. However, this set may contain redundant information by containing several time-frequency locations belonging to a single spectral window.

To refine the signal detection, we keep only the local maxima along the frequencies of the selected coefficients, in order to select only the maxima of the spectral windows. Local maxima along the time is not considered, given that stationary frequencies does not present local maxima along time.

The set of local maxima $\mathcal{M}$ is

$$
\begin{gathered}
\mathcal{M}=\left\{[n, k] / D[n, k] \geq D\left[n, k^{\prime}\right]\right\}, \\
k^{\prime}=[k-10, k-9, \ldots k+9, k+10]
\end{gathered}
$$

The range of frequencies around $k$, from $k-10$ to $k+10$ has been chosen large enough to avoid selecting too many local maxima, and small enough to include close different frequencies.

Finally, the set of time-frequency coefficients $\mathcal{P}$ used in the chirp estimation is the intersection of the set of detected signal points and the set of local maxima

$$
\mathcal{P}=\mathcal{S} \cap \mathcal{M}
$$

The selected coefficients are gathered in the next section, and used to estimate the chirps parameters. 


\section{CHIRPS ESTIMATION}

Given the set of chirplet coefficients detected in the previous section, we aim to gather them in a small set of chirps in a real time framework, these chirps corresponding to each linear part of the analysed signal. Each chirp is characterised by four parameters: the starting point $n_{s}$, the end point $n_{e}$, the initial frequency $k_{i}$ at time $n_{s}$ and the chirprate $c r$.

For all selected chirplet coefficients, from the smallest time index and frequency index to the highest indices, we search if the chirplet coefficients fits in an existing chirp, and update the chirp's parameters with the new coefficient. Otherwise, we create a new chirp, whose starting point and end point are the first point and the last point of the window associated with the chirplet coefficient, chirprate is set to the chirplet's chirprate, and initial frequency is deduced from the central frequency and the chirprate of the chirplet. The algorithm is summarised as follows:

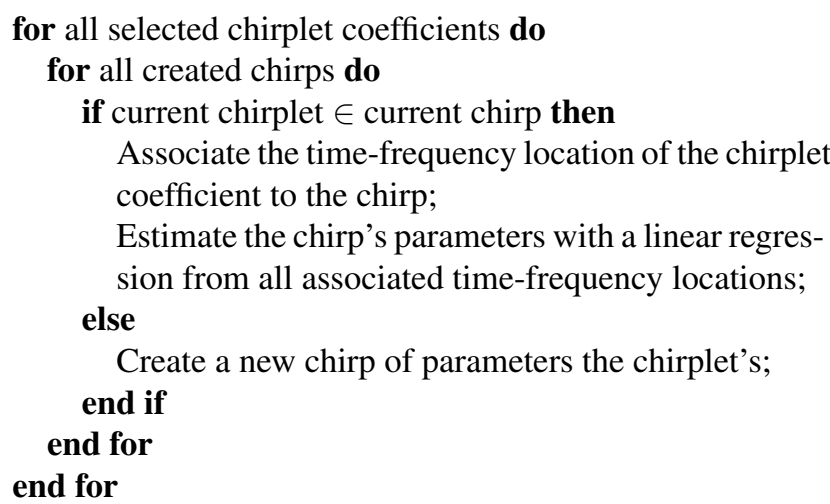

The conditions for the belonging of a chirplet coefficient, centred on time index $n$ and frequency index $k$ with a chirprate $c$, to a chirp are:

- time condition, the chirp and the chirplet overlap:

$$
n_{s} \leq n \leq n_{e}+\delta_{t} \text {; }
$$

- chirprate condition, the chirplet and the chirps have approximately the same chirprate:

$$
|c-c r| \leq \delta_{c} .
$$

- frequency condition, the central frequency of the chirplet is located on the chirp:

$$
\left|k_{i}+\operatorname{cr}\left(n-n_{i}\right)-k\right| \leq \delta_{k} ;
$$

Each selected chirplet is associated to only one chirp. In other words, chirplets located at the crossing of two chirps can be linked to only one of these chirps, and the other chirp will miss this coefficient. Moreover, the detection step may miss some signal coefficients. Consequently, the gathering process should be tolerant enough to miss some coefficients.

The three thresholds $\delta_{t}, \delta_{k}$ and $\delta_{c}$ are chosen in respect to the parameters of the chirplet transform. The time threshold $\delta_{t}$ allows the association of closely disconnected chirplets to a chirp. In the following, we set $\delta_{t}=M_{\phi}$, the length of the analysis window of the chirplet transform.

The chirprate threshold $\delta_{c}$ is set to the chirprate discretization step and the frequency threshold $\delta_{k}$ to the maximum between the frequency index step and the frequency index step due to the discretization of the chirprate

$$
\begin{aligned}
\delta_{c} & =\frac{1}{L} c_{\max } \\
\delta_{k} & =\max \left(1, K \frac{l}{L} c_{\max } \frac{M_{\phi}}{2}\right)
\end{aligned}
$$

When chirps are crossing, some chirplet coefficients may be wrongly selected due to the interferences between the chirps. As a post-processing, we remove chirps with length equal to the length of a single chirplet.

\subsection{Results}

Figure 1 shows the result of the algorithm on a synthetic signal made of 20 chirps, with a SNR of $3 \mathrm{~dB}$. The chirplet is computed with a Gaussian window of 4095 points, an overlap between consecutive windows of 2047 points, and 4096 computed frequencies. 51 discrete chirprates are computed, from $-10^{-5}$ to $10^{-5}$.

98 chirps are created from 379 selected chirplets coefficients. The post-processing removes 78 chirps made of only one chirplet, leading to the 20 chirps existing in the analysed signal.

The errors on the estimated starting points and end points are all smaller than the overlap parameter of the chirplet transform, and the error of the estimated frequency smaller than the frequency step. Thanks to the linear regression, the chirprates are very accurately estimated: the errors on the chirprates vary from $1 / 5$ to $1 / 40$ of the chirprate step of the chirplet transform, except for a chirp with an error equal to approximately the chirprate step.

The most computationally intensive part of the full analysis is the chirplet transform, which takes twice the computational time of the chirplet selection and the chirps estimation.

\section{CONCLUSION}

We have presented in this paper an efficient algorithm for detecting and estimating linear chirps, in a context of LPI radars made of frequency modulation waves. Using the chirplet transform, a first step selects a few relevant chirplet coefficients, containing the signal and being local maxima along frequencies. This step avoids including redundant chirplet coefficients. The second step gathers these coefficients with criterion based on time, frequency and chirprate, and estimates the chirps from a linear regression from the gathered coefficients. A post-processing removes "false alarm" chirps created with wrongly selected coefficients in the first step. This algorithm has been illustrated on a synthetic signal imitating $4 \mathrm{FMCW}$ radars. 

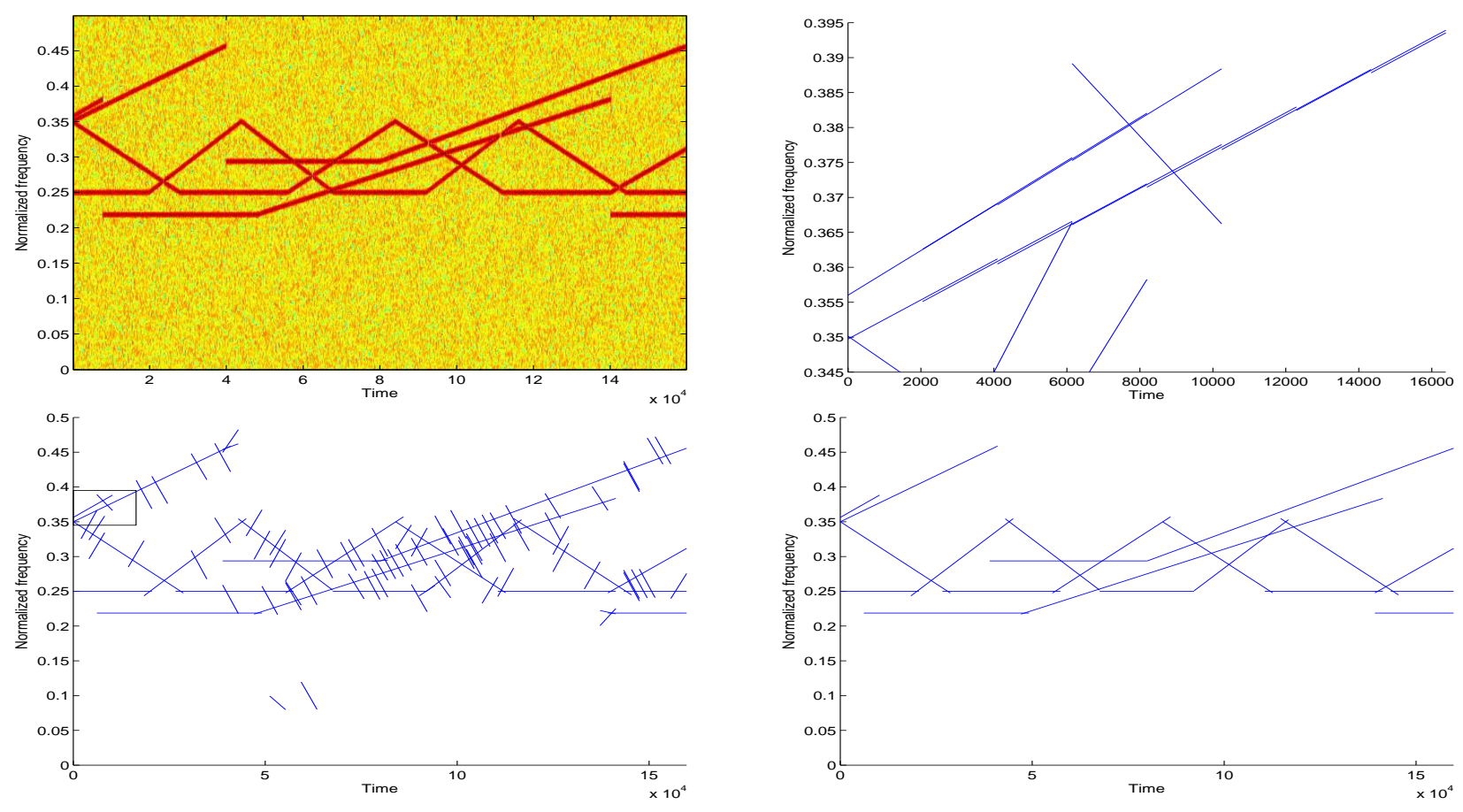

Fig. 1. Up left, spectrogram of the synthetic signal to analyse. Up right, zoom on the select chirplets. Down left, results of the gathering of selected chirplets, with a Gaussian windows of $M_{\phi}=4095$ points. Short "false alarm" chirps are created where frequencies are crossing, as well as at beginnings and ends of chirps. The black box indicates the area of the zoom of the up right figure. Down right, the estimated chirps without the false-alarm chirps removed by the post-precessing.

The result on a synthetic signal are very good and accurately estimates the chirps parameters. The next step is to use these results in order to classify the radars. This may be done by gathering the obtained chirps in order to estimate their modulation bandwidths, modulation periods and carrier frequencies. Another approach may be to use dictionaries of FMCW radars, searching for the different pulse repetitions and chirprates.

Future work will consider the influence of the parameters and try to determine the minimal redundancy of the chirplet transform required for the algorithm to give good results.

\section{REFERENCES}

[1] Aytug Denk, Detection and jamming low probability of intercept (LPI) radars, Ph.D. thesis, Naval Postgraduate School, 2006.

[2] S. Mann S. and Haykin S., "The chirplet transform: A generalization of gabor's logon transform," Calgary, Canada, June 1991.

[3] P. Leveau, L. Daudet, S. Krstulović, and R. Gribonval, "Model-based matching pursuit - estimation of chirp factors and scale of gabor atoms with iterative extension," in Signal Processing with Adaptative Sparse Structured Representations, Rennes, France, Nov. 2005.

[4] A. Pai, E. Chassande-Mottin, and O. Rabaste, "Best network chirplet chain: Near-optimal coherent detection of unmodeled gravitational wave chirps with a network of detectors," Phys. Rev. D, vol. 77, no. 062005, pp. 1-22, 2008.

[5] Emmanuel J. Candès, Philip R. Charlton, and Hannes Helgason, "Detecting highly oscillatory signals by chirplet path pursuit.," Appl. Comput. Harmon. Anal., vol. 24, no. 1, pp. 14-40, 2008.

[6] B. Dugnol, C. Fernández, G. Galiano, and J. Velasco, "On a chirplet transform-based method applied to separating and counting wolf howls," Signal Process., vol. 88, no. 7, pp. 1817-1826, 2008.

[7] George Casella and Roger L. Berger, Statistical Inference, Duxbury Resource Center, 2nd edition, 1993.

[8] F. Millioz, , and N. Martin, "Estimation of a white Gaussian noise in the Short Time Fourier Transform based on the spectral kurtosis of the minimal statistics: application to underwater noise," in Proceedings of IEEE Conference on Acoustics, Speech and Signal Processing, Dallas, Texas, USA, Mar. 2010. 